\title{
Rencontre avec Stéphane Braunschweig
}

\section{(e) OpenEdition}

1 Journals

\section{Édition électronique}

URL : http://journals.openedition.org/shakespeare/1306

DOI : 10.4000/shakespeare.1306

ISSN : 2271-6424

Éditeur

Société Française Shakespeare

\section{Édition imprimée}

Date de publication : 1 novembre 1995

Pagination : 165-173

\section{Référence électronique}

"Rencontre avec Stéphane Braunschweig 》, Actes des congrès de la Société française Shakespeare [En ligne], 13 | 1995, mis en ligne le 01 janvier 2007, consulté le 29 avril 2019. URL : http://

journals.openedition.org/shakespeare/1306 ; DOI : 10.4000/shakespeare.1306 


\title{
SHAKESPEARE \\ LE MONDE VERT : RITES ET RENOUVEAU
}

Société Française Shakespeare

\author{
Actes du Congrès 1994 \\ sous la direction \\ de \\ M.T. JONES-DAVIES
}

Ouvrage publié avec le soutien de

BARCLAYS

BARCLAYS BANK S.A.

PARIS

LES BELLES LETTRES

1995 
Tous droits de traduction, de reproduction et d'adaptation réservés pour tous les pays.
(C) 1995 Société d'édition Les Belles Lettres, 95 bd Raspail 75006 Paris 


\section{Rencontre avec Stéphane Braunschweig}

\section{directeur du Théâtre d'Orléans}

D.G-B. : S. Braunschweig, merci d'être venu. Vous dirigez le Conte d'hiver qui passe en ce moment au Théâtre de Gennevilliers. $\mathrm{S}$. Braunschweig n'a plus besoin d'être présenté. Il a fait une entrée très remarquée sur la scène parisienne avec La Trilogie des hommes de neige qui était l'aboutissement d'un travail de plusieurs années. Il y a eu ensuite une Cerisaie assez provocante, un Faust qui viendra à Paris à l'automne.

Votre spectacle dure quatre heures, qui passent très vite. C'est le texte de Shakespeare sans coupure. C'est aussi une question de rythme que vous avez choisi d'adopter pour arriver à la gravité de la scène finale, très belle et très recueillie.

S.B. : Le Conte d'hiver est l'une des pièces les plus longues. Il est normal que la représentation dure longtemps. Il y a quelques coupures de détail effectuées pour des raisons techniques, ... je n'avais pas douze satyrs pour une danse, à deux cela aurait paru grotesque. On a aussi éliminé quelques redites. On ne peut pas jouer Shakespeare en français avec la même rapidité qu'en anglais, et je ne sais pas si cela est souhaitable. Autant quand j'avais monté Tchekhov j'avais essayé de jouer très vite : La Cerisaie tenait en deux heures. Dans le cas du Conte je ne m'étais pas donné de contrainte de temps. On n'a pas cherché à créér des rythmes, on a essayé de faire un tout organique. Il faut cependant noter que la pastorale est plus rapide que le reste. Le temps n'est pas non plus étiré, il n'y a pas de temps psychologique, ou du temps inutile qui nous donnerait à penser qu'il y a constamment du sous-texte. Cette longueur vise à faire entendre le texte le mieux possible. Mais la question de la longueur est totalement subjective : on peut ne pas s'ennuyer pendant huit heures de spectacle. Mais pourquoi s'ennuie-t-on? Parce que la place du spectateur n'a pas été dessinée.

Dans Le Conte, le spectacle vient percuter le regard du spectateur. Lè regard est l'un des thèmes majeurs de la pièce.

D.G-B.: Vous avez fait jouer le personnage du Temps par Hermione enceinte, au moment où l'histoire va accoucher de sa suite. 
S.B. Je reviens toujours à des questions matérielles. Je ne pouvais pas engager un acteur juste pour faire le Temps. Shakespeare aussi avec une troupe réduite. Je voulais une femme, et celle qui disparaît dans la pièce, celle qui est à la fois mère, grosse d'un avenir, et en même temps qui est profondément liée à la mort. Le Temps est l'ambivalence entre la mort et la maternité. Il me semblait évident que l'actrice qui joue Hermione, dans son costume, doive dire ce texte. "S'il permet votre patience ..." Le spectacle dure déjà depuis deux heures. Ce n'est pas un parti-pris allégorique et pompier. C'est de l'ordre du récit théâtral avec une dimension humoristique. L'actrice ne joue pas cette partie comme elle joue Hermione.

D.G-B. : Nous avons été nombreux à trouver très beau et en même temps très astucieux l'utilisation que vous faites de l'espace et du dispositif scénique.

S.B. : C'est le dispositif scénique qui, lui, crée des contraintes. C'est un plateau d'abord plat et en forte pente (45\%) vers le fond de la scène. C'est très difficile pour les acteurs de tenir, mais c'est la limite praticable. C'était une intuition de départ de vouloir travailler sur un espace vertigineux. Un tel espace bouleverse le jeu des acteurs, redétermine la relation entre les corps, ils peuvent exister. On est obligé de se concentrer : un instant de déconcentration, et c'est la chute. A vrai dire, on ne chute jamais, mais on est toujours au bord de la chute : c'est bien le thème du Conte. Ainsi en pente, ce lieu projette l'histoire, la fable, les personnages vers les spectateurs, donc les spectateurs ne sont pas à la recherche de ce qui se passe sur le plateau, c'est nous qui allons vers eux, à la rencontre de leur regard.

Si l'on veut essayer de comprendre la différence entre la partie tragique et la partie comique, on est obligé de se rendre compte que le regard du spectateur change de nature. Dans un premier temps, le spectateur n'en sait pas plus que les personnages, il travaille à la conviction, non à la preuve. Toute l'ambiguïté est de savoir si la jalousie de Leontes a un fondement ou si c'est de la folie. Shakespeare se garde bien de nous donner des éléments de réponse qui aillent dans un sens ou un autre. Petit à petit, le spectateur peut se forger une conviction, du fait qu'on a toujours envie d'être du côté des victimes, cette pulsion-là l'emporte peut être pour un instant. La vérité, établie par l'oracle, à la fin de cette par- 
tie-ci, vient donner une certitude au spectateur. L'espace tragique est ainsi créé.

Dans la deuxième partie, le spectateur sait tout. Il a même le pressentiment que l'action va bien finir sur le plateau. Certaines thématiques qui sont filées dans la première partie sont reprises dans la deuxième : la tyrannie des pères, la bâtardise. Sachant tout, le spectateur a une puissance presque voyeuriste. Tous les personnages ont une part de vérité tellement tronquée que cela crée des situations selon un principe de comédie bien connu. La deuxième partie traite de l'erreur. Puisqu'on ne peut pas établir de vérité, vautrons-nous dans le mensonges : Autolycus qui raconte les ballades fausses, on ne peut pas croire à cette histoire de poisson apparu le 30 avril sur la côte à 40.000 brasses au-dessus de l'eau. Il y a une complicité entre tous les personnages dans cette jouissance du mensonge, c'est la scène emblématique de cette partie.

Les deux attitudes — se vautrer dans le mensonge, être à la recherche d'une vérité objective - , procèdent d'une même illusion que Shakespeare démasque, sur un mode tragique pour l'une, sur un mode comique pour l'autre. Le regard que l'on peut porter sur le monde est idéologiquement fermé. La fin de la pièce me semble ouvrir sur un rapport en phase avec le monde, on va au réél, on sort de la fiction.

D.G-B. : Pendant le monologue de Leontes, Polyxenes a la main posée sur le ventre d'Hermione. Par un jeu d'éclairage subtil on voit ce qu'imagine Leontes. Un spectateur peu averti pourrait comprendre que Polyxenes réclame la paternité de cet enfant, qu'il n'y a pas beaucoup de doute sur l'inconduite de la reine.

S.B. : L'ambiguïté est terrible : c'est celle qui existe entre le désir et le passage à l'acte. Le texte montre que Polyxenes a du désir pour Hermione quand il prend la défense de la reine avec excès. Lorsqu'il fuit à la fin de l'acte I en disant qu'elle est pure, je ne pense pas qu'il y ait d'innocence. En évoquant son enfance avec Leontes, Polyxenes décrit bien le monde de l'innocence : un jardin d'Eden où ils étaient sans la femme, sans le désir. Lorsqu' Hermione le questionne sur le désir, sur la tentation, Polyxenes répond : "A ce moment-là, il n'y avait pas les femmes, ni de tentation, ni vous. "La femme de Polyxenes qui est évoquée n'est jamais là, si elle était présente, il ne se passerait rien. On est dans une situation de déséquilibre : une femme, deux hommes. La relation qui lie la femme à Leontes n'est pas plus forte que celle qui unit Leontes à 
Polyxenes. Qui est l'intrus dans ce trio ? La réponse change constamment.

La jalousie de Leontes se déclanche de façon brutale, arbitraire, mais elle n'est pas entièrement infondée. Shakespeare donne tous les moyens de comprendre ce mécanisme, mais il n'en fait pas le descriptif psychologique. Cette pièce ne traite pas de la montée de la jalousie, Shakespeare ne refait pas Othello. En revanche, il y a un fondement. Et cette relation qui unit Polyxenes à Leontes est aussi une relation d'amour.

Le jeu de lumière qui pose l'ambiguïté au moment où Leontes dit: "Trop chaud, trop chaud!" peut aussi être compris comme le fantasme de Leontes. Une zone de lumière plus forte arrive sur lui, le quatrième mur du théâtre semble s'ouvrir vers les spectateurs.

Ce qui est montré entre Polyxenes et Hermione peut-être crédible sans pour autant qu'il y ait de la part d'Hermione conscience d'être en situation de séduction. Polyxenes a conscience du désir qu'il peut avoir pour Hermione, c'est la raison de son départ; il fuit sans donner d'argument. Hermione n'a pas conscience de l'effet qu'elle peut produire. C'est son drame, elle ne se comprend pas elle-même.

Jean-Michel DÉPRATS: Je me sens indirectement coupable d'avoir nourri ton analyse sur la lecture fantasmatique. J'admire la finesse de ton analyse sans être convaincu, mais j'admire la mise en scène et cette séquence en particulier. J'étais très irrité par la mise en scène anglaise qui montrait d'une manière crue, voire vulgaire, une relation de flirt explicite entre Hermione et Polyxenes avec des effets de ralentissements qui levaient toute ambiguïté, même si par ailleurs il n'y avait aucune forme de violence, ni jalouse ni tragique, dans le jeu de l'acteur. Je ne crois pas qu'il y ait d'ambiguïté possible quant à la vérité même si Shakespeare crée une situation dans laquelle les affects s'entrelacent de telle manière que cette irruption n'est pas irrationnelle, brutale sans doute, mais non psychologique.

Grâce à cet effet de lumière nous était donné à voir à la fois la situation que tu as décrite comme une séduction réciproque non consciente et la projection du fantasme de Leontes. On n'était pas du tout dans le cas de figure où on postulerait comme impossible un amour entre Polyxenes et Hermione. On était plutôt dans les 
données de l'inconscient avec la projection d'un fantasme de Leontes.

S.B. : Dans le texte, Leontes dit : "Se tripoter les mains comme ils sont en train de le faire". Je n'ai aucune raison de penser qu'il s'agit d'un fantasme.

Henry SUHAMY : Il est clairement dit dans le texte qu'il s'agit du fantasme de Leontes dans le monologue très décousu de l'acte $I$, scène 2. C'est l'imagination qui le crée, à partir du néant, en se fondant sur la passion. Il y a là une analyse subtile du mécanisme du fantasme.

J-M.D. : Ne s'agit-il pas là du mécanisme du désir ?

H.S. : Désir et fantasme se nourrissent aux mêmes sources.

S.B. : Je ne conteste pas qu'il y a fantasme de Leontes. Au moment où cela est dit, on ne le sait pas On ne peut pas passer son temps a relire la pièce en commençant par la fin.

HS : On sent la jalousie à plein nez.

SB : $\quad$ Sur le plateau, ils ne se "tripotent" pas les mains, ils se prennent les mains, et se caressent. Je ne satisfait pas le fantasme de Leontes. Oui, ils se prennent par la main, Leontes, lui, dit qu'ils se les tripotent.

QU : Je voudrais que l'on revienne sur le regard. Il y a un espace discontinu qui oblige le spectateur à modifier continuellement sa position - donc il ne s'agit pas que des acteurs. C'est un dispositif pictural qui condense non seulement les corps, mais les paroles. Dans ce dispositif s'inscrit un trou, au sens strict. Donc on donne déjà à voir sur plusieurs niveaux, puisqu'un autre epace est possible, l'espace d'enfermement. Cet espace entraîne une parfaite lisibilité entre Hermione vivante et morte. Le fantasme n'est pas encore mort, tout reste dans une ambiguïté absolue, ni la vérité, ni la fiction, entre la vie et la mort, la mort et la vie.

S.B. : La fin n'est pas claire. Comme principe déontologique, je ne vais pas faire semblant de comprendre, peut-être ne faut-il pas comprendre.

La mise en scène essie de se situer à un certain niveau pulsionnel, et non pas au niveau du conscient. Elle nous laisse en permanence en prise avec l'ambiguitté, l'ambivalence, et même la non-réalisation des désirs. L'espace ne peut être que mental et non 
réaliste. J'essaie de ne pas réduire l'interprétation. C'est autant une réduction interprétative que d'affirmer que Polyxenes et Hermione sont innocents de tout désir que de dire qu'ils sont coupables.

Il faudrait revenir sur le rôle que joue l'enfant, il assiste à toute la scène, puis il disparàit pour revenir dans la deuxième partie. Il y a une sorte de gemellité au niveau des deux rois, qui se retrouve, au niveau des deux enfants réciproques de ces rois. Le fait que l'un des deux soit absent est aussi un déséquilibre structurel. D'une certaine manière, l'arrivée d'un deuxième enfant, de la maternité, est un déséquilibre fondateur qui va faire naître le désir de Polyxenes pour Hermione.

La même question se pose à la fin : comment ne pas réduire la scène? Shakespeare donne des éléments concrets sur la résurrection. Il nous laisse bien à penser que ce n'est pas une vraie - et en même temps, c'est une vraie résurrection. Du point de vue technique, cette scène n'est pas difficile à réaliser. Il suffit de mettre un acteur au milieu sans bouger, puis il se met en mouvement. On n'a pas besoin de statue, ni de costume, ni de maquillage. Au theâtre on croît à tout, si l'on nous dit d'y croire. C'est bien les paroles de Paulina: "Si vous êtres capables de regarder, éveillez votre foi !" C'est la question de la croyance dans ce que l'on va voir. Shakespeare ouvre totalement cet espace.

Plus j'ai avancé dans le travail, plus j'ai pensé qu'il y avait absence de dénouement. Dans un premier temps les seigneurs racontent ce qui s'est passé hors-scène, cette séquence prépare la scène de la résurrection. Mais le dénouement important a lieu hors scène. Celui qui est empreint de fiction, de comédie n'a aucune importance. Le seul intérêt du dénouement est ce que l'on peut en dire, une fin heureuse est-elle de la joie ou de la souffiance? Lorsque l'on raconte un conte à un enfant, on veut lui épargner "la réalité du réel" selon le terme de Gargani, dans Regard et Destin. Mais l'enfant sait très bien que le réel ne correspond pas à ce qu'on lui dit, où est la joie, où est la souffrance, dans l'illusion ou dans la vérité ? On a donc une impossibilité d'interpréter le dénouement. Une fois que cet axiome est posé, on peut entrer dans la scène de la résurrection, qui est elle-même une scène d'ouverture :

"Conduis nous loin d'ici pour qu'on puisse s'interroger

Sur le rôle que chacun de nous a joué

Dans ce vaste abîme du temps." 
Cette scène évide le théâtre de l'intérieur, et creuse la place du spectateur. Jusqu'à présent, il se trouvait en position frontale par rapport au spectacle, on lui a donné une lisibilité. Dans cette scène, on ne lui donne plus à voir, c'est une suspension du voir, qui relie à la question de la mort. Dans la première partie, on voit le travail de la mort, la deuxième partie est le refoulement de la mort, dans la troisième, on dépasse complètement cette dialectique. On a bien un personnage qui revient d'entre les morts - ou qui n'en revient pas - , mais qui vient nous confronter à l'expérience de la perte. Ce n'est pas par hasard si cela correspond à la fin du spectacle, il y a une certaine tristesse de la fin de la représentation.

H.S. : Je voulais simplement rappeler, comme on le sait, que la jalousie est un thème obsessionnel dans le théâtre shakespearien. C'est un thème tragique, celui du déchirement, de la séparation, fondé sur l'ambiguiité même de l'amour, puisque la jalousie, c'est l'amour et la haine qui se confondent. Lorsque vous lisez Beaucoup de bruit pour rien, Les Joyeuses commères de Windsor, Othello, Cymberline, toutes ces pièces qui utilisent le même scénario que dans Le Conte d'hiver c'est-à-dire le thème de la jalousie infondée, appliquez-vous la même analyse ? Shakespeare a brodé plusieurs fois sur ce même thème.

S.B. : N'étant pas spécialiste de Shakespeare, je n'ai aucune analyse précise sur ces œuvres-là. Dans Othello, il y a le personnage de Iago. Il n'existe pas de personnage semblable dans $L e$ Conte.

H.S. : Il est intériorisé chez Leontes. C'est un thème qui a été traité fréquemment au cours des siècles.

S.B. : Intériorisé ? L'ambition de Iago, la vengence de Iago ?

HS : Le langage obscène de Leontes est une espèce d'intériorisation de Iago. On a intérêt à se pencher sur les pièces précédentes pour voir comment le thème évolue, l'auteur creuse sa propre pensée. Iago devient Iachimo dans Cymberline, et disparaît complètement dans Le Conte. Il disparaît physiquement, mais pas idéologiquement.

S.B. : Ce texte qui a été traduit par :

"Désir, ton intensité frappe au centre,

Tu rends possible des choses tenues pour impossibles." 
Shakespeare dit bien que l'on n'a pas besoin de Iago. C'est peut-être la chose nouvelle, non pas l'ambiguïté de l'amour, mais l'ambivalence du désir.

François Laroque: Vous maintenez votre tempo grâce à l'ampleur de la musique. Travaillez vous la musique de la voix, au détriment des corps ?

S.B. : Il n'y a pas de travail vocal particulier. Mais je suis toujours attentif à la qualité de la voix.

F.L. : Votre Leontes n'est pas vociférant, mais presque grinçant.

SB : $\quad$ Cela ne me plaît pas de postuler que Leontes est fou, de même que cela ne m'intéresserait pas de partir de l'idée que Lear est fou, ou Hamlet, parce qu'on n'est pas dans la tragédie grecque, les personnages n'ont pas une hubris terrible. Ce sont des humains, vous, moi, qui peuvent devenir des monstres. C'est le "devenir monstre" qui est intéressant. Ajax, que j'ai monté, est exactement la problématique inverse, c'est l'histoire du "devenir homme". Il y a chez Shakespeare l'idée que tout le monde peut devenir fou, personne n'est à l'abri, et de même, il n'y a pas une nature de fou.

Je ne vais pas donner une image vociférante de Leontes, ce serait un cliché. Mais voir comme la folie est aussi une forme de raison, extrêmement élaborée.

La pièce a aussi un intérêt politique : comment une histoire individuelle, intime, affective, devient politique. Parce que Leontes est roi, cette histoire est exposée en public.

F.L. : A propos de l'intervalle, nous avons été surpris de la place inhabituelle de l'entracte.

S.B. : A partir du moment où l'on pense que cette pièce peut contenir une troisième partie, on repense tout autrement. Si l'on coupe entre les deux pièces, on va effectivement donner l'impression qu'il y a deux pièces en une. Or la deuxième commence sur les ruines de la première, avec les thématiques qui vont ressortir. Je voulais que la première partie ne s'arrête pas sur la révélation de la mort de la reine. Chez Shakespeare il y toujours cette nécessité d'enchaîner, de continuer. On ne peut pas s'en tenir à cette pastorale. Autolycus est le premier personnage qui intervient dans cette deuxième partie ainsi créée. Il y a une justification matérielle à l'entracte : "Nous allons nous déguiser". On prend dix minutes 
pour aller se déguiser, et on va rentrer dans "son" théâtre, c'est lui le personnage emblématique, dans ce divertissement commercial où tous les personnages vont être déversés. Dans la deuxième partie, deux histoires se télescopent, celle d'Autolycus, et l'histoire d'amour Florizel-Perdita. C'est aussi l'expression d'une certaine vision du monde. La dernière phrase de l'acte IV est prononcée par Autolycus : "Qui sait comment tout cela va rebondir pour favoriser mon avancement". Ce personnage est très individualiste, il parle tout le temps d'argent, voilà pourquoi j'ai centré sur lui.

Margaret JONES-DAVIES : J'ai beaucoup aimé votre mise en scène. Au début, on a une vision très nette du texte matérialisé dans des objets. En revanche, ce grand drap rouge n'a pas de signification précise au début. Pourtant, à la fin, avec la dualité d'Hermione qui change de regard, ce tissu rouge représente l'ordre social.

S.B. : Ce rideau rouge est le rideau d'un petit théâtre qui se trouve au fond et qui dégouline sur l'avant. En étant installés dessus, les personnages n'avaient pas conscience d'être au théâtre, que le drame était en train de se jouer sous leurs pieds. Que le rideau revienne à la fin, couvrant la statue, montre que la résurrection, c'est du théâtre, et en même temps, c'est l'endroit où ce théâtre s'annule.

D.G-B. : Il est malheureusement temps de se quitter. Ceux qui n'ont pas encore vu le spectacle vont s'y précipiter après vous avoir entendu. Nous vous avons écouté avec grand plaisir et on a vu la pièce avec beaucoup d'émotion. Merci.

Texte transcrit par Mme Isabelle Schwartz-Gastine 